\title{
Prognostic significance of an apoptotic index and apoptosis/proliferation ratio for patients with high-grade astrocytomas ${ }^{1}$
}

Hiroko Kuriyama, Kathleen R. Lamborn, Judith R. O'Fallon, N. Iturria, Thomas Sebo, Paul L. Schaefer, Bernd W. Scheithauer, Jan C. Buckner, Nagato Kuriyama, Robert B. Jenkins, and Mark A. Israel ${ }^{2}$

The Preuss Laboratory for Molecular Neuro-Oncology, Brain Tumor Research Center (H.K., N.K., M.A.I.); Department of Neurological Surgery, University of California, San Francisco, CA 94143 (H.K., K.R.L., N.K., M.A.I.); Mayo Clinic and Foundation, Rochester, MN 55905 (J.R.O., N.I., T.S., B.W.S., J.C.B., R.B.J.); Toledo Community Hospital Oncology Program, Toledo, OH 43623 (P.L.S.)

We evaluated the association of spontaneous apoptosis and an apoptosis/proliferation index with survival to determine the potential of such measures to serve as predictive markers for patients with glioblastoma multiforme (GBM). We examined the extent of spontaneous apoptosis in tumors from newly diagnosed patients, 75 with GBM and 21 with anaplastic astrocytoma, who were entered on treatment protocols of the North Central Cancer Treatment Group. In the group of GBM patients, those with a higher apoptotic index tended to live longer $(P=0.04$; Cox proportional hazards model including performance score, age, and extent of resection in a multivariate model). We found that the apoptotic index values for anaplastic astrocytoma patients tended to be lower than those in the GBM patients, although with small sample sizes, the result was not statistically significant

Received 23 July 2001, accepted 2 April 2002.

${ }^{1}$ This work was supported in part by CA64898 (M.A.I., K.R.L.), CA82103 (K.R.L.), CA50905 (J.R.O., T.S., J.C.B., R.B.J.), and CA 25224 (J.R.O., N.I., P.L.S., B.W.S., J.C.B.) and the Anne and Jason Farber Foundation (M.A.I.), the Betz Foundation (M.A.I.), and the Jordan and Kyra Memorial Fund (M.A.I.).

${ }^{2}$ Address correspondence and reprint requests to Mark A. Israel, Norris Cotton Cancer Center, Dartmouth Medical School, Dartmouth-Hitchcock Medical Center, 1 Medical Center Dr., Lebanon, NH 03756.

${ }^{3}$ Abbreviations used are as follows: $\mathrm{AA}$, anaplastic astrocytoma; $\mathrm{Al}$, apoptotic index; GBM, glioblastoma multiforme.
$(P=0.1)$. We also examined expression of the Ki-67 cell proliferation antigen immunohistochemically using the MIB-1 monoclonal antibody. Ki-67 expression did not provide additional information regarding the survival of patients with GBM. In this group of GBM patients, those patients with higher apoptotic index/proliferation ratios had a better prognosis than did those with a low ratio $(P<$ 0.021 , same model as above). These findings suggest that both apoptosis and a cell death/cell proliferation ratio are associated with patient survival, and they may be useful for either the clinical evaluation of patients with GBM or the stratification of patients for treatment evaluation. Neuro-Oncology 4, 179-186, 2002 (Posted to NeuroOncology [serial online], Doc. 01-042, May 23, 2002. URL <neuro-oncology.mc.duke.edu>)

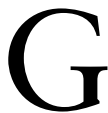
liomas are the most common primary brain tumors of both children and adults. Among those tumors, GBM, ${ }^{3}$ a highly malignant astrocytic tumor, is particularly resistant to current antineoplastic strategies such as radiation and chemotherapy. There are no widely recognized or validated biologic markers predictive of outcome for patients with this tumor. This limits both the ability of clinicians to optimally prognosticate and the ability of clinical researchers to effectively stratify patients for therapy.

One possible prognostic marker for patients with malignant tumors may be apoptosis, and this possibility has been tested in patients with several tumor types (Chung et al., 1999; Kawauchi et al., 2000; Komaki et 
al., 1996; Leoncini et al., 1993; Levine et al., 1995; Lipponen et al., 1994; Macluskey et al., 2000; Tanaka et al., 1999; Xie et al., 1999). Spontaneous apoptosis is a common finding in biopsy specimens of many cancers (Gorczyca et al., 1994), including some types of brain tumors (Kordek et al., 1996; Migheli et al., 1994; Patsouris et al., 1996). The inability of tumor cells to undergo apoptosis may be an important mechanism of resistance to current antineoplastic strategies, and the degree of apoptosis observed in tumor specimens obtained prior to the initiation of cytotoxic therapy might predict prognosis, especially in tumors generally resistant to therapy (Desoize, 1994). Previous studies have shown that the amount of apoptosis detectable in tumors correlates with patient survival for patients with various glial tumors (Korshunov et al., 1999; Schiffer et al., 1997), although other studies have not detected such an association (Haapasalo et al., 1999; Kordek et al., 1996; Nakamura et al., 1997; Wharton et al., 1998).

Other possible biologic markers helpful for the management of brain tumor patients include measures of cell proliferation (Cunningham et al., 1997; Haapasalo et al., 1999; Heesters et al., 1999; Nakamura et al., 1997; Wharton et al., 1998). Several groups have previously examined both apoptosis and proliferation in brain tumors (Heesters et al., 1999; Schiffer et al., 1995; Wharton et al., 1998), and these studies provide data suggesting that markers of cellular proliferation and apoptosis increase with increasing grade of malignancy in astrocytic tumors. Rhodes examined 39 astrocytomas of several grades and observed a tendency for cases with less proliferation than apoptosis to have a more favorable outcome (Rhodes, 1998). The prognostic significance of a ratio of apoptosis to proliferation has also been examined in other cancers. A high ratio of apoptosis to proliferation was found to correlate with improved survival and to be a better prognostic marker than the amount of apoptosis alone in cervical adenocarcinoma and colorectal cancer (Koike, 1996; Sheridan et al., 1999). A 2.5 -fold increase of the mitosis/apoptotic index was observed in pathologic specimens from ductal carcinoma in situ of the breast compared to specimens from poorly differentiated invasive breast carcinoma (Mommers et al., 1999).

In this study, we examined spontaneous apoptosis by an in situ end-labeling technique and cellular proliferation by immunohistochemical staining of the Ki-67 cell proliferation antigen in tumors from 96 newly diagnosed, carefully monitored, protocol-treated patients with high-grade astrocytic tumors (75 GBMs, 21 AAs). We assessed the association of apoptosis and the ratio of apoptosis to proliferation with the survival of these protocol-treated patients.

\section{Methods}

\section{Patient Population}

We examined tumor specimens from 75 patients diagnosed with GBM and 21 patients diagnosed with AA at the time of their initial presentation. All specimens were from tumor tissue obtained at the time of initial surgery and were determined to be high-grade glioma. At presentation, each of these patients was entered into one of 9 prospective clinical trials conducted by the Mayo Clinic and the North Central Cancer Treatment Group between June 20, 1985, and December 26, 1997. All 9 trials were conducted on newly diagnosed high-grade glioma patients whose tumors were centrally reviewed by experienced neuropathologists. None of the patients had received prior cytotoxic or radiation therapy. All patients were treated with radiation therapy and nitrosoureabased chemotherapy after surgery. Follow-up was available on all patients entered into these clinical trials and was not a selection criterion for this study.

The 96 specimens we examined were drawn from a collection of 174 high-grade astrocytoma specimens (111 GBMs, 63 AAs) from patients in 10 clinical trials (the 9 above plus an earlier trial) that were assembled circa 1995 for glioma marker research purposes. The Glioma Markers Tissue Collection (North Central Cancer Treatment Group study 947252) contained all AA specimens then available from all North Central Cancer Treatment Group institutions and all GBM specimens available for the Mayo Clinic patients in these trials. Of those, only 75 GBM and 21 AA specimens were of sufficient size for the determination of an $\mathrm{AI}$ as described below.

To look for differences between the patients who were and were not in our study, we compared, separately for AAs and GBMs, the clinical characteristics and survival of our 96 study patients with those of the 78 patients (42 AA, $36 \mathrm{GBM}$ ) with specimens in the Glioma Markers Tissue Collection that were too small for our study, as well as with those of the 1125 patients (90 AAs, 1035 GBMs) in the 10 clinical trials but whose specimens were not in the Glioma Markers Collection. The patients in our study were similar to the others in age, sex, and performance score, but differed significantly in extent of resection. Because of the need for adequate tissue, our patients tended to have more extensive resections than the others and, therefore, may have had a slightly better prognosis. There were also fewer patients older than 60 years in the group we studied than in the other groups $(10 \%$ versus $25 \%-31 \%$ patients with AA, $32 \%$ versus $43 \%-50 \%$ patients with GBMs). Nonetheless, the survival distributions of our patients were very similar to those of the other Glioma Markers Collection patients $(\log$-rank $P$ values $=0.94$ and 0.83 for those with AA and for those with GBM, respectively).

\section{Evaluation of Apoptosis}

Brain tumor specimens were formalin fixed and then paraffin embedded. After hematoxylin- and eosinstained tumor sections were examined to confirm the histopathology of individual specimens, sections adjacent to those evaluated were prepared for further analysis. Histologic sections on glass slides were heated for $5 \mathrm{~min}$ in a microwave oven (Quasar; Thomas, Swedesboro, N.J.) at $370 \mathrm{~W}$ and incubated at $65^{\circ} \mathrm{C}$ for $1.5 \mathrm{~h}$. Deparaffinization was completed by incubation in xylene and rehydration in a series of graded ethanol concentrations. Apoptosis was detected by an in situ endlabeling technique that recognizes DNA breaks resulting 
from endonuclease activity (ApopTag Plus; Intergen, Purchase, N.Y.). Diaminobenzidine was used to detect apoptotic cells, which stained dark brown. Control sections were evaluated simultaneously, substituting distilled water for active enzyme. All slides were counterstained with hematoxylin.

For this study, we defined an AI as the average number of apoptotic cells in 20 blindly selected high-power fields in a single histologic tumor section from each patient. A Leica DMLS microscope with an attached grid was used to count the cells at a magnification $\times 400$. The risk category and the clinical status of each patient were unknown to laboratory investigators. We designated specimens as suitable for determination of an $\mathrm{AI}$ if there was sufficient tumor to allow counting 20 high-power fields, while avoiding the necrotic areas and the edge of the tissue.

\section{Evaluation of Proliferation}

Histologic sections were deparaffinized and used for immunohistochemical analysis of Ki-67 with the MIB-1 monoclonal antibody (Cunningham et al., 1997). The MIB-1 labeling index was determined with a digital analyzer as previously described (Sebo, 1995). The MIB-1 indices used in this study were the percentage of labeled nuclei in the same tumor specimen employed to determine the AI. No attempt was made to determine MIB-1 on sections immediately sequential to the sections employed for the evaluation of AI. The MIB-1 scoring method used and the evaluation of these specimens have been previously described (Cunningham et al., 1997).

\section{Statistical Methods}

Based on our experience with this apoptosis assay, we determined that the assay should be considered semiquantitative and that use of the actual numbers represented spurious precision. The AI values were categorized as low, moderate, or high depending on whether the AI was $<0.5,0.5$ to 1.5 , or $>1.5$. In fact, there were few values near the cut points of the categories.

To compare tumor grades for AI scores, we used the Wilcoxon test with the exact permutation distribution. Survival from date of entry into the clinical trial was the primary end point of that study. Time-to-progression information was not felt to be sufficiently reliable to evaluate. The primary method for analyzing survival was the Cox proportional hazards regression. This method was selected because it allowed evaluation of $\mathrm{AI}$ as a prognostic factor, taking into account clinical variables known or thought to predict survival time. Specifically, we considered age at diagnosis; performance status measured by Eastern Cooperative Oncology Group criteria; and extent of resection measured as biopsy, subtotal resection, or gross total resection. Extent of resection was determined by both the surgeon's report and the postoperative scans on these patients treated under protocol.

While evaluating MIB-1 reactivity, we noted that very large values were occasionally observed. Because proportional hazards models give disproportionate weight to large values, we classified the MIB-1 values in ordered categories for analysis. Because we had no prior informa- tion on which to base the selection of cut points, we used a scoring system based on the quartiles of the MIB-1 score of 67 patients for whom we were able to obtain MIB-1 data. The quartile scores were 1 (MIB- $1<6.8$ ), 2 $(6.8 \leq$ MIB-1 < 14.1), 3 (14.1 $\leq$ MIB-1 < 20.5), and 4 (MIB-1 $\geq 20.5$ ). For analysis, we created an AI/MIB-1 score with the ratio of the AI index (scored as 1 for low, 2 for moderate, and 3 for high, as defined above) and the MIB-1 score (scored as 1, 2, 3, or 4 based on the quartile designation). Use of the scores in a Cox proportional hazards model assumes that the hazard ratio for a given incremental change between scores is the same across the range of the data. Although this might not be precisely true, the scores, rather than indicator values, were used in the analyses to take into account the ordering from low to high. Although no formal testing was done to evaluate this assumption, apparent deviations are addressed in "Results." We did not use raw data for AI or MIB for the reasons stated above. We do not believe such a strategy would introduce bias, because the categories were selected without knowledge of outcome. For display purposes, Kaplan-Meier survival curves were used. Because there were too many AI/MIB-1 categories to readily display a separate curve for each, patients were combined into 3 groups based on low, moderate, and high AI/MIB ratios to provide approximately equal numbers of patients in each group. The precise number in each group was determined by the number of patients who had values at the cut points and thereby had to be included together in a single group. The analyses described above are those for which results are reported. Additional analyses to test the robustness of the assumptions, including the use of indicator variables, tests for interactions, and review of residuals, were conducted. Because these additional tests raised no major issues with the originally planned analyses, they are not discussed further. The AI/ MIB ratio was used instead of the 2 variables as potentially independent predictors in a single proportional hazards model because others have reported the data in this manner. Again, use of the more traditional model did not change the overall conclusions and so is not specifically discussed in "Results."

\section{Results}

\section{Distribution of AI Based on Clinical Characteristics}

Table 1 shows the distribution of apoptosis in patients with GBM and AA. Forty percent of GBM tumor specimens could be placed in the group that had an AI of $>0.5$, whereas $19 \%$ of AA tumor specimens were in this same range. Because of the small number of AA cases, there was low power to detect differences between the 2 histology groups, and the trend toward lower AI scores in the AA group was not statistically significant using the exact Wilcoxon rank sum test $(P=0.1)$.

The clinical characteristics of the 75 patients with GBM included in our study are shown in Table 2. The age distribution in this study was similar to other studies with larger numbers of GBM patients. Sex distribution in this study was 1:2 (F:M) compared with 1:1.5 in most 
Table 1. Distribution of apoptosis index in patients with GBM and AA

\begin{tabular}{|c|c|c|c|c|c|c|}
\hline \multirow[b]{2}{*}{ Al group } & \multirow[b]{2}{*}{ Score } & \multirow[b]{2}{*}{$\mathrm{Al}$} & \multicolumn{2}{|c|}{ GBM } & \multicolumn{2}{|c|}{$\mathrm{AA}$} \\
\hline & & & No. of patients & $\%$ & No. of patients & $\%$ \\
\hline Low & 1 & $<0.5$ & 45 & 60 & 17 & 81 \\
\hline Moderate & 2 & $0.5-1.5$ & 23 & 31 & 3 & 14 \\
\hline High & 3 & $>1.5$ & 7 & 9 & 1 & 5 \\
\hline
\end{tabular}

Table 2. Clinical characteristics of 75 patients with GBM

\begin{tabular}{lc} 
Factor & No. of patients (\%) \\
\hline Age & $6(8)$ \\
$\quad<40$ & $45(60)$ \\
$40-60$ & $24(32)$ \\
$>60$ & \\
Sex & $51(68)$ \\
$\quad$ Male & $24(32)$ \\
Female & \\
Baseline performance score & $58(77)$ \\
$0-1$ & $17(23)$ \\
$2-4$ & \\
Extent of surgical resection & $10(13)$ \\
Biopsy & $46(61)$ \\
Subtotal & $19(25)$ \\
Gross total & \\
\hline
\end{tabular}

studies of GBM patients. This, however, does not influence our survival analysis, because sex is not a prognostic factor for the survival of GBM patients (Ganju et al., 1994). Because of the need for adequate tissue, our study included fewer biopsied patients than might be expected.

\section{Prediction of Survival}

We analyzed patients with GBM for correlates of survival. Two patients were alive at the time of analysis and had been followed for 587 and 717 days from entry on clinical trial. There were too few patients with a diagnosis of AA to study survival prediction in that group. We examined the relationship between survival and AI, MIB1 score, age, performance status, and extent of resection. We found an association between age and survival as one would expect in a GBM population (Shrieve et al., 1999). We also found an association between AI and survival, but no other associations were detected. Kaplan-Meier survival curves for GBM patient groups, defined by AI, are shown in Fig. 1 and indicate that higher AI is predictive of better survival. To determine if this finding added information to the known prognostic characteristics of GBM patients, we used Cox proportional hazards modeling to adjust for performance score, age, and extent of resection in a multivariate model. We found that, based on tumor AI, the hazard ratio for patients with GBM was 0.67 with a $95 \%$ confidence interval of 0.46 to 0.98 and $P=0.04$. This hazard ratio predicts that when the amount of apoptosis in a pretreatment biopsy specimen of GBM increases sufficiently to move tumors from one level of apoptosis to the next, the patient's risk decreases by approximately one third. Recognizing that there are only 7 patients in the high apoptosis group and that the survival of these patients was similar to that in the group with moderate apoptosis (Fig. 1), we believe the primary determinant of this hazard ratio is the difference in survival between the groups with low as compared to moderate apoptosis.

The tumor pathology might be heavily dependent on the balance of cell death and cell proliferation, and previous studies have suggested a relationship between the

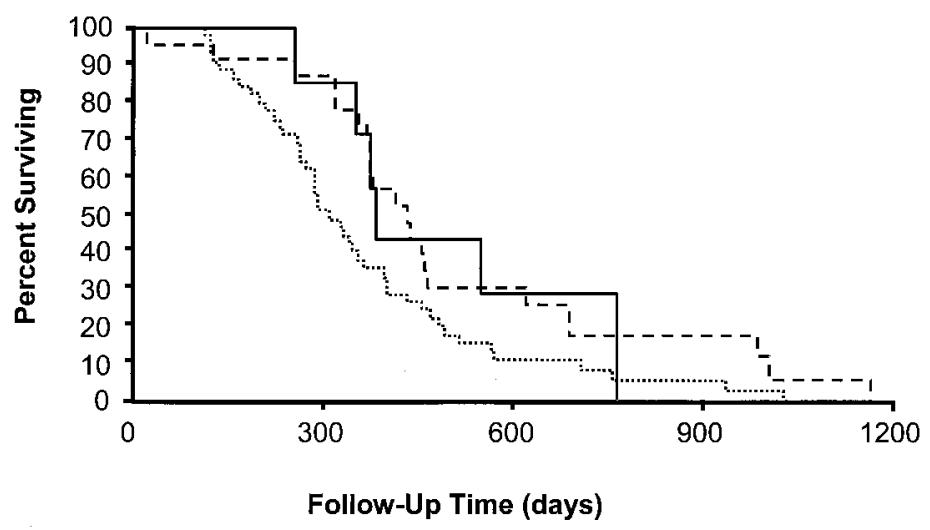

Fig. 1. Kaplan-Meier survival curves for patients with GBM whose tumors exhibited low, moderate, or high Al. Curves represent the survival of patients with low $\mathrm{Al}(<0.5, n=45$, dotted line), moderate $\mathrm{Al}(0.5-1.5, n=23$, dashed line), and high $\mathrm{Al}$ ( $\mathrm{Al}>1.5, n=7$, solid line). Median survivals for low, moderate, and high Al groups were 311 (95\% Cl, 275-366), 435 (95\% Cl, 373-466), and 384 (95\% Cl, 351-769) days, respectively. Abbreviation: $\mathrm{Cl}$, confidence interval. 


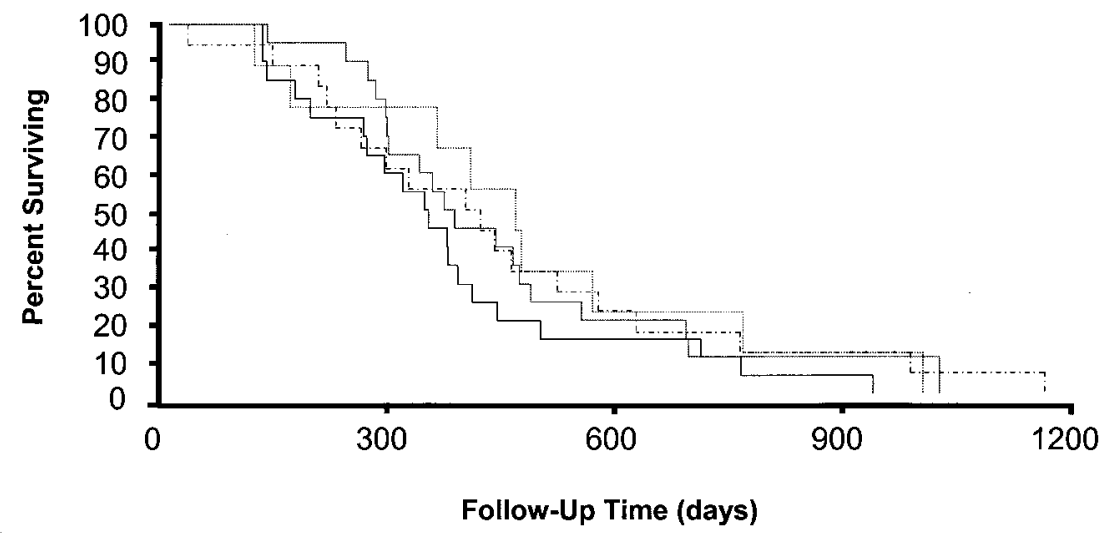

Fig. 2. Kaplan-Meier survival curves for patients with GBM divided into 4 groups based on MIB-1 labeling scores as described in "Methods": MIB-1 <6.8 ( $n=9$, dotted line); $<6.8$ to $<14.1$ ( $n=18$, dashed/dotted line); $<14.1$ to $<20.5$ ( $n=20$, solid black line); and MIB-1 > 20.5 $(n=20$, gray line).

outcome of brain tumor patients and various measures of tumor cell proliferation (Grzybicki and Moore, 1999). Therefore, we evaluated MIB-1 reactivity, a marker of tumor cell proliferation, in these same tumor specimens. The MIB-1 score in patients with GBM was significantly higher than it was in patients with AA $(P<0.0012)$ (data not shown). In contrast to the finding of an association between survival and AI, we detected no similar association between MIB-1 and survival of GBM patients using the proportional hazards model for MIB-1 (using same model as above, hazard ratio for MIB-1, 1.17; $P=0.21$ ). The Kaplan-Meier curves relating the MIB-1 score to survival are shown in Fig. 2. No evidence of a correlation between survival and MIB-1 expression is apparent.

We then sought to examine if there was an association between the ratio of $\mathrm{AI}$ to the MIB-1 score (AI/MIB-1) with survival using the proportional hazards model. We found that the hazard ratio for AI/MIB-1 with survival was 0.51 (95\% confidence interval 0.29-0.90; $P=0.021$; using same model as above). For display purposes, we placed patients into 3 approximately equally sized groups based on AI/MIB-1 scores. The Kaplan-Meier survival curves for patient groups defined by the AI/MIB-1 ratio (Fig. 3) demonstrate that patients with the highest ratios had the longest survival and showed patterns of survival similar to those observed in Fig. 1. These displays, together with the statistical analyses, including an analysis adding the AI/MIB ratio to a model including $\mathrm{AI}$ and the clinical variables, indicate that no substantial improvements in the potential for prognostication were realized by using the AI/MIB- 1 ratio in contrast or in addition to $\mathrm{AI}$ alone to categorize patients.

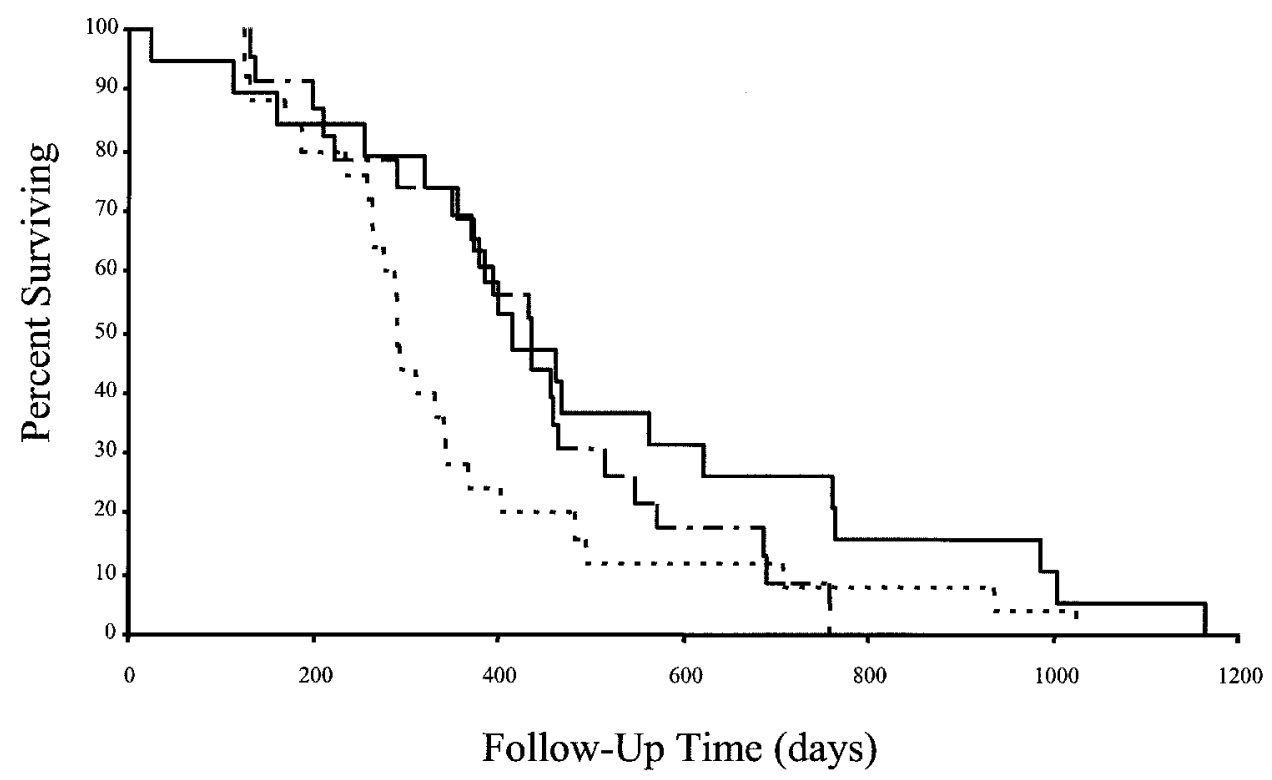

Fig. 3. Kaplan-Meier survival curves for patients with GBM in 3 groups categorized by an AI/MIB-1 ratio. Curves represent the survival of patients with a low Al/MIB-1 ratio $(<0.5, n=25$, dotted line), a moderate ratio $(<0.5$ to $<1.0, n=23$, dashed/dotted line), or a high ratio $(>1.0, n=19$, solid line). Median survivals for low, moderate, and high Al/MIB-1 ratio groups were 290 (95\% Cl, 263-345), 435 (95\% Cl, 370-466), and 414 (95\% Cl, 357-622) days, respectively. 
Although the statistical analysis does indicate that $\mathrm{AI}$ alone and the AI/MIB-1 ratio each provide some information on the prognosis of patients as a group, as can be readily ascertained by review of Figs. 1 and 3, there is still substantial variation in outcome among patients with similar scores. To better describe the extent to which these measures would accurately predict the survival of individual patients, we compared the AI and AI/MIB-1 values for the one third of patients who died first and the one third who survived the longest. Because a few patients did not have MIB-1 values determined, the actual number of patients for whom we were able to determine an AI/MIB1 ratio were 22 (early deaths) and 25 (late deaths). Looking first at AI, we noted that of the 22 early deaths, 19 were in the lowest AI group and 3 were in the intermediate AI group. One additional patient for whom MIB-1 was not available was in the highest AI group. However, 11 patients in the longest surviving group of 25 patients also had AIs that would have placed them in the poorest prognosis group. These findings suggest that, although high AI was a good sign, it was not totally predictive of good survival. Some patients with low AI still did well. A similar pattern can be seen in looking at AI/MBI-1 ratios. Among the poor outcome patients, 12 had AI/MIB-1 ratios of .25 or .33, implying that they both had very low AI and were in one of the highest two MIB-1 groups, which would predict their poor outcome. Six patients had an AI/MIB-1 ratio of .5 , and 4 had a ratio of 1 . With these higher ratios, the 10 poor outcome patients would have been predicted to be among the longer-term survivors if the AI/ MIB-1 were used as the predictor. Among the longer survivors, 5 had AI/MIB- 1 ratios of $<0.5,11$ had intermediate AI/MIB- 1 ratios, and 9 had ratios of $\geq 1$. Clearly, the AI/MIB-1 ratio provides some information about prediction, but it also misclassifies a number of patients, as would the use of AI alone.

\section{Discussion}

Astrocytic tumors of the CNS are an important clinical problem, and the development of successful strategies for their prevention and treatment has been elusive. Among astrocytic tumors, high-grade tumors, including AA and GBM, directly invade and grow in brain tissue, frequently recur at the site of the original tumor, and almost invariably are the cause of death for patients in whom they arise. Our inability to properly classify such tumors as homogeneous clinical entities may contribute to the difficulties in developing effective treatments. The identification of such tumor groupings would greatly facilitate our ability to conduct effective clinical research and should enhance our ability to provide a more accurate prognosis as well. No tumor markers are currently recognized to be reliable indicators of prognosis for patients with astrocytic tumors, and none are widely used in the management of these patients. Clinical predictors of prognosis for patients with astrocytic tumors include tumor grade, patient age, and performance status.

We have evaluated the potential of two biologically relevant measures to classify patients with GBM treated on a series of comparable clinical protocols. Because
GBM is an intracranial tumor and relatively resistant to all available modalities of therapy, we thought it might be a tumor in which patient outcome would be closely related to tumor growth. For this reason, we examined both a measure of cell death and the ratio of cell death to cell proliferation, important variables contributing to tumor growth. Proliferation was evaluated by measuring the extent of Ki-67 expression: a nuclear protein that is present in all phases of the cell cycle but is not expressed when cells are in $\mathrm{G}_{0}$. To estimate the proportion of tumor cells undergoing apoptosis, we used an assay that measures the labeling of DNA breaks. To avoid artifacts associated with the edge of the tissue and necrotic areas, we determined an AI only on specimens from tumors that had sufficient tissue for these areas to be avoided during the analysis.

Proliferation markers have been widely studied in numerous tumor types. In most studies of brain tumors, these have been found to be associated with pathologic tumor grade, but any association with prognosis within grade, if there is one, is much less clear (for review, see Grzybicki and Moore, 1999). Apoptosis also has been examined previously in pretreatment specimens of patients with a variety of tumors including brain tumors. High levels of apoptosis may predict a good outcome for patients with squamous cell carcinoma of the lung, prostate, and tongue, non-small cell lung cancer, and Wilms tumor (Komaki et al., 1996; Macluskey et al., 2000; Tanaka et al., 1999; Wheeler et al., 1995; Xie et al., 1999), but a poor survival in patients with cervical carcinoma, synovial sarcoma, breast cancer, nonHodgkin lymphoma, and others (Kawauchi et al., 2000; Leoncini et al., 1993; Levine et al., 1995; Lipponen et al., 1994). Although these studies generally evaluated rather small numbers of patients, they suggest that the prognostic value of apoptosis might be tumor specific.

Several groups have reported that the increasing pathologic grade of glial CNS tumors is associated with an increase in apoptosis (Ellison et al., 1995; Heesters et al., 1999; Kordek et al., 1996; Schiffer et al., 1995). Other studies have sought an association between apoptosis and the clinical outcome of patients with these tumors. In specimens of both GBM and medulloblastoma, high levels of apoptosis are related to a better patient prognosis (Haslam et al., 1998; Korshunov et al., 1999). Schiffer et al. (1997) found that high levels of apoptosis correlate weakly with poor survival for patients with oligodendroglioma. Other studies have not detected an association between clinical outcome and apoptosis in patients with oligodendroglioma (Heesters et al., 1999; Wharton et al., 1998), pilocytic astrocytoma (Haapasalo et al., 1999), anaplastic astrocytoma (Heesters et al., 1999; Nakamura et al., 1997), or GBM (Heesters et al., 1999). None of these studies evaluated patients treated on protocol.

We evaluated specimens for evidence of apoptosis from patients treated under protocol for AA or GBM. As has been found in previous studies, we observed high levels of apoptosis to occur more frequently in tumor tissue from GBM patients than in tissue from AA patients (Table 1) (Ellison et al., 1995; Heesters et al., 1999; Kordek et al., 1996; Schiffer et al., 1995). This finding is enigmatic because GBM is generally considered a more 
malignant tumor than AA; however, there are multiple determinants of tumor growth that might contribute to this apparent association. For example, past studies have generally reported more extensive proliferation in GBM than in AA (Grzybicki and Moore 1999). This would further support the concept that the ratio of AI to proliferation might be most predictive.

There were sufficient numbers of patients with GBM examined in this study to seek a correlation between survival and apoptosis. We found that high levels of apoptosis were related to an increased duration of survival in this group of patients. This result implies that the low levels of spontaneous apoptosis in pretreatment tumor specimens may contribute to more rapid tumor growth or, if spontaneous apoptosis is a predictor of the capacity of a tissue to undergo DNA damage-induced apoptosis, low levels of spontaneous apoptosis might correlate with enhanced resistance to cytotoxic treatment strategies. In most tumor types, apoptosis is thought to contribute significantly to the response of tumors to these modalities (Kaufmann and Earnshaw, 2000). Although all patients we evaluated received both radiation and chemotherapy, there is considerable evidence suggesting that, in GBM tissues, the apoptotic response to these modalities is quite limited (Haas-Kogan et al., 1996; Weller et al., 1998). It seems unlikely, therefore, that the amount of apoptosis in these biopsy specimens is a marker of therapeutic response.

To explore the potential importance of the balance between cellular proliferation and apoptosis in predicting clinical outcome, we sought to determine whether the association of AI with survival could be enhanced by combining AI with an index of tumor cell proliferation, MIB-1, in these tumors. Several studies have previously assessed the prognostic significance of a ratio of apoptosis to proliferation in various tumor types including gliomas (Koike, 1996; Mommers et al., 1999; Rhodes,
1998; Schiffer et al., 1995; Sheridan et al., 1999). A high ratio of apoptosis to proliferation was found to correlate with improved survival and a better prognosis in patients with cervical adenocarcinoma (Sheridan et al., 1999). In this study, the ratio of apoptosis to proliferation was found to be a better predictor of prognosis than AI alone (Sheridan et al., 1999). Rhodes (1998) examined 39 astrocytomas of several grades and observed that patients whose tumors had less proliferation than apoptosis tended to have a more favorable outcome. We found in our group of 75 GBM patients treated on protocol that lower AI/MIB-1 ratios were predictive of shorter survival (Fig. 3). However, the association of this ratio and survival was not substantially better than the association of AI alone to survival (Fig. 1). This finding suggests that despite the low level of apoptosis found in GBM tissue specimens, and despite the lack of extensive apoptosis after the treatment of GBM-derived tissue with DNA damaging agents (Haas-Kogan et al., 1996; Weller et al., 1998), the extent of apoptosis itself or biologic alterations closely associated with apoptosis may mark a group of tumors that are biologically distinct (Fisher 1994). It is also possible that there are host defenses not currently recognizable that are reflected in the extent of tumor apoptosis. In either case, the amount of apoptosis in pretreatment biopsy specimens is associated with the survival of patients with GBM and may enhance our ability to more effectively group patients for evaluation in clinical trials, and if refined further, may help with determining prognosis for individual patients.

\section{Acknowledgment}

The authors thank Mae Joy de la Calzada for her editorial assistance.

\section{References}

Chung, E.J., Seong, J., Yang, W.I., Park, T.K., Kim, J.W., Suh, C.O., and Kim, G.E. (1999) Spontaneous apoptosis as a predictor of radiotherapy in patients with stage IIB squamous cell carcinoma of the uterine cervix. Acta Oncol. 38, 449-454.

Cunningham, J.M., Kimmel, D.W., Scheithauer, B.W., O'Fallon, J.R., Novotny, P.J., and Jenkins, R.B. (1997) Analysis of proliferation markers and p53 expression in gliomas of astrocytic origin: Relationships and prognostic value. J. Neurosurg. 86, 121-130.

Desoize, B. (1994) Anticancer drug resistance and inhibition of apoptosis. Anticancer Res. 14, 2291-2294.

Ellison, D.W., Steart, P.V., Gatter, K.C., and Weller, R.O. (1995) Apoptosis in cerebral astrocytic tumours and its relationship to expression of the bcl-2 and p53 proteins. Neuropathol. Appl. Neurobiol. 21, 352-361.

Fisher, D.E. (1994) Apoptosis in cancer therapy: Crossing the threshold. Cell 78, 539-542

Ganju, V., Jenkins, R.B., O'Fallon, J.R., Scheithauer, B.W., Ransom, D.T., Katzmann, J.A., and Kimmel, D.W. (1994) Prognostic factors in gliomas: A multivariate analysis of clinical, pathologic, flow cytometric, cytogenetic, and molecular markers. Cancer 74, 920-927.

Gorczyca, W., Tuziak, T., Kram, A., Melamed, M.R., and Darzynkiewicz, Z. (1994) Detection of apoptosis-associated DNA strand breaks in fineneedle aspiration biopsies by in situ end labeling of fragmented DNA. Cytometry 15, 169-175
Grzybicki, D.M., and Moore, S.A. (1999) Implications of prognostic markers in brain tumors. Clin. Lab. Med. 19, 833-847.

Haapasalo, H., Sallinen, S., Sallinen, P., Helen, P., Jaaskelainen, J., Salmi, T.T., Paetau, A., Paljarvi, L., Visakorpi, T., and Kalimo, H. (1999) Clinicopathological correlation of cell proliferation, apoptosis and p53 in cerebellar pilocytic astrocytomas. Neuropathol. Appl. Neurobiol. 25, 134-142.

Haas-Kogan, D.A., Yount, G., Haas, M., Levi, D., Kogan, S.S., Hu, L., Vidair, C., Deen, D.F., Dewey, W.C., and Israel, M.A. (1996) p53-dependent G1 arrest and $\mathrm{p} 53$-independent apoptosis influence the radiobiologic response of glioblastoma. Int. J. Radiat. Oncol. Biol. Phys. 36, 95-103.

Haslam, R.H., Lamborn, K.R., Becker, L.E., and Israel, M.A. (1998) Tumor cell apoptosis present at diagnosis may predict treatment outcome for patients with medulloblastoma. J. Pediatr. Hematol. Oncol. 20, 520-527.

Heesters, M.A., Koudstaal, J., Go, K.G., and Molenaar, W.M. (1999) Analysis of proliferation and apoptosis in brain gliomas: Prognostic and clinical value. J. Neurooncol. 44, 255-266.

Kaufmann, S.H., and Earnshaw, W.C. (2000) Induction of apoptosis by cancer chemotherapy. Exp. Cell Res. 256, 42-49.

Kawauchi, S., Fukuda, T., Oda, Y., Saito, T., Oga, A., Takeshita, M., Yokoyama, K., Chuman, H., Iwamoto, Y., Sasaki, K., and Tsuneyoshi, M. (2000) Prognostic significance of apoptosis in synovial sarcoma: Correlation with clinicopathologic parameters, cell proliferative activity, and expression of 
apoptosis-related proteins. Mod. Pathol. 13, 755-765.

Koike, M. (1996) Significance of spontaneous apoptosis during colorectal tumorigenesis. J. Surg. Oncol. Suppl. 62, 97-108.

Komaki, R., Fujii, T., Perkins, P., Ro, J.Y., Allen, P.K., Mason, K.A., Mountain, C.F., and Milas, L. (1996) Apoptosis and mitosis as prognostic factors in pathologically staged N1 nonsmall cell lung cancer. Int. J. Radiat. Oncol. Biol. Phys. 36, 601-605.

Kordek, R., Hironishi, M., Liberski, P.P., Yanagihara, R., and Gajdusek, D.C. (1996) Apoptosis in glial tumors as determined by in situ nonradioactive labeling of DNA breaks. Acta Neuropathol. (Berl) 91, 112-116.

Korshunov, A., Golanov, A., Sycheva, R., and Pronin, I. (1999) Prognostic value of tumour associated antigen immunoreactivity and apoptosis in cerebra glioblastomas: An analysis of 168 cases. J. Clin. Pathol. 52, 574-580.

Leoncini, L., Del Vecchio, M.T., Megha, T., Barbini, P., Galieni, P., Pileri, S., Sabattini, E., Gherlinzoni, F., Tosi, P., Kraft, R. [AU: list all authors] (1993) Correlations between apoptotic and proliferative indices in malignantnonHodgkin's lymphomas. Am. J. Pathol. 142, 755-763.

Levine, E.L., Renehan, A., Gossiel, R., Davidson, S.E., Roberts, S.A., Chadwick, C., Wilks, D.P., Potten, C.S., Hendry, J.H., Hunter, R.D. [AU: list all authors] (1995) Apoptosis, intrinsic radiosensitivity and prediction of radiotherapy response in cervical carcinoma. Radiother. Oncol. 37, 1-9.

Lipponen, P., Aaltomaa, S., Kosma, V.M., and Syrjanen, K. (1994) Apoptosis in breast cancer as related to histopathological characteristics and prognosis. Eur. J. Cancer 30A, 2068-2073.

Macluskey, M., Baillie, R., Chandrachud, L.M., Pendleton, N., and Schor, A.M. (2000) High levels of apoptosis are associated with improved survival in non-small cell lung cancer. Anticancer Res. 20, 2123-2128.

Migheli, A., Cavalla, P., Marino, S., and Schiffer, D. (1994) A study of apoptosis in normal and pathologic nervous tissue after in situ end-labeling of DNA strand breaks. J. Neuropathol. Exp. Neurol. 53, 606-616.

Mommers, E.C., van Diest, P.J., Leonhart, A.M., Meijer, C.J., and Baak, J.P. (1999) Balance of cell proliferation and apoptosis in breast carcinogenesis. Breast Cancer Res. Treat. 58, 163-169.

Nakamura, M., Konishi, N., Tsunoda, S., Hiasa, Y., Tsuzuki, T., Inui, T., and Sakaki, T. (1997) Retinoblastoma protein expression and MIB-1 correlate with survival of patients with malignant astrocytoma. Cancer 80, 242-249.

Patsouris, E., Davaki, P., Kapranos, N., Davaris, P., and Papageorgiou, K. (1996) A study of apoptosis in brain tumors by in situ end-labeling method. Clin. Neuropathol. 15, 337-341.

Rhodes, R.H. (1998) Biological evaluation of biopsies from adult cerebral astrocytomas: Cell-growth/cell-suicide ratios and their relationship to patient survival. J. Neuropathol. Exp. Neurol. 57, 746-757.

Schiffer, D., Cavalla, P., Migheli, A., Chio, A., Giordana, M.T., Marino, S., and Attanasio, A. (1995) Apoptosis and cell proliferation in human neuroepithelial tumors. Neurosci. Lett. 195, 81-84.

Schiffer, D., Dutto, A., Cavalla, P., Chio, A., Migheli, A., and Piva, R. (1997) Role of apoptosis in the prognosis of oligodendrogliomas. Neurochem. Int. 31, 245-250.

Sebo, T.J. (1995) Digital image analysis. Mayo Clin. Proc. 70, 81-82.

Sheridan, M.T., Cooper, R.A., and West, C.M. (1999) A high ratio of apoptosis to proliferation correlates with improved survival after radiotherapy for cervical adenocarcinoma. Int. J. Radiat. Oncol. Biol. Phys. 44, 507512.

Shrieve, D.C., Alexander, E., III, Black, P.M., Wen, P.Y., Fine, H.A., Kooy, H.M., and Loeffler, J.S. (1999) Treatment of patients with primary glioblastoma multiforme with standard postoperative radiotherapy and radiosurgical boost: Prognostic factors and long-term outcome. J. Neurosurg. 90, 72-77.

Tanaka, K., Granata, C., Wang, Y., O'Briain, D.S., and Puri, P. (1999) Apoptosis and bcl-2 oncogene expression in Wilms' tumor. Pediatr. Surg. Int. 15 , 243-247.

Weller, M., Rieger, J., Grimmel, C., Van Meir, E.G., De Tribolet, N., Krajewski, S., Reed, J.C., von Deimling, A., and Dichgans, J. (1998) Predicting chemoresistance in human malignant glioma cells: The role of molecular genetic analyses. Int. J. Cancer 79, 640-644.

Wharton, S.B., Hamilton, F.A., Chan, W.K., Chan, K.K., and Anderson, J.R. (1998) Proliferation and cell death in oligodendrogliomas. Neuropathol. Appl. Neurobiol. 24, 21-28.

Wheeler, J.A., Stephens, L.C., Tornos, C., Eifel, P.J., Ang, K.K., Milas, L., Allen, P.K., and Meyn, R.E., Jr. (1995) ASTRO Research Fellowship: Apoptosis as a predictor of tumor response to radiation in stage IB cervical carcinoma. American Society for Therapeutic Radiology and Oncology. Int. J. Radiat. Oncol. Biol. Phys. 32, 1487-1493.

Xie, X., De Angelis, P., Clausen, O.P., and Boysen, M. (1999) Prognostic significance of proliferative and apoptotic markers in oral tongue squamous cell carcinomas. Oral Oncol. 35, 502-509. 